\title{
Loss of CSMD1 expression disrupts mammary duct formation while enhancing proliferation, migration and invasion
}

\author{
MOHAMED KAMAL ${ }^{1,2}$, DEBORAH L. HOLLIDAY ${ }^{3}$, EWAN E. MORRISON ${ }^{1}$, \\ VALERIE SPEIRS $^{3}$, CARMEL TOOMES $^{1}$ and SANDRA M. BELL ${ }^{1}$ \\ ${ }^{1}$ Leeds Institute of Biomedical and Clinical Sciences, University of Leeds, Wellcome Trust Brenner Building, \\ St. James's University Hospital, Leeds LS9 7TF, UK; ${ }^{2}$ Department of Zoology, Faculty of Science, \\ University of Benha, Benha 13518, Egypt; ${ }^{3}$ Leeds Institute of Cancer and Pathology, University of Leeds, \\ Wellcome Trust Brenner Building, St. James's University Hospital, Leeds LS9 7TF, UK
}

Received August 17, 2016; Accepted May 11, 2017

DOI: 10.3892/or.2017.5656

\begin{abstract}
The CUB and sushi multiple domains 1 (CSMD1) gene maps to chromosome 8 p23, a region deleted in many cancers. Loss of CSMD1 expression is associated with poor prognosis in breast cancer suggesting that it acts as a tumour suppressor in this cancer. However, the function of CSMD1 is largely unknown. Herein, we investigated CSMD1 functions in cell line models. CSMD1 expression was suppressed in MCF10A and LNCaP cells using short hairpin RNA. Functional assays were performed focusing on the 'normal' MCF10A cell line. Suppression of CSMD1 significantly increased the proliferation, cell migration and invasiveness of MCF10A cells compared to shcontrols. shCSMD1 cells also showed significantly reduced adhesion to Matrigel and fibronectin. In a three-dimensional Matrigel model of MCF10A cells, reduced CSMD1 expression resulted in the development of larger and more poorly differentiated breast acini-like structures that displayed impaired lumen formation. Loss of CSMD1 expression disrupts a model of mammary duct formation while enhancing proliferation, migration and invasion. Our data suggest that CSMD1 is involved in the suppression of a transformed phenotype.
\end{abstract}

Correspondence to: Dr Sandra M. Bell, Leeds Institute of Biomedical and Clinical Sciences, University of Leeds, Wellcome Trust Brenner Building, St. James's University Hospital, Beckett Street, Leeds LS9 7TF, UK

E-mail: medsmb@leeds.ac.uk

Abbreviations: CDCP1, CUB domain containing protein 1; CSMD1, CUB and sushi multiple domains 1; CSMD2, CUB and sushi multiple domains 2; CSMD3, CUB and sushi multiple domains 3; ECM, extracellular matrix; FCS, foetal calf serum; IHC, immunohistochemistry; MTT, 3-(4,5-dimethylthiazol-2-yl)2,5-diphenyltetrazolium bromide; qRT-PCR, quantitative real-time polymerase chain reaction; RPLP0, 60S acidic ribosomal protein P0; RT-PCR, reverse transcriptase polymerase chain reaction; SFM, serum-free medium; shRNA, short hairpin RNA

Key words: CSMD1, proliferation, migration, invasion, MCF10A 3D model, breast cancer

\section{Introduction}

CUB and sushi multiple domains-1 (CSMD1) is a very large gene which contains 71 exons that span over $2 \mathrm{Mb}$ of genomic DNA on chromosome 8p23 (1). Multiple splice variants exist for CSMD1 and these encode proteins of varying length. The largest transcript is $14.3 \mathrm{~kb}$ long and this encodes a 3,565 amino acid protein (1). The full-length protein is a membrane protein with an extracellular region containing 14 CUB and 28 sushi domains, a single transmembrane domain and a short cytoplasmic domain that contains a putative tyrosine phosphorylation site. CSMD1 belongs to the CSMD gene family whose members also include the structurally similar proteins CSMD2 and CSMD3 (1-3). The function of CSMD1 is largely unknown. Although CSMD1 has been shown to inhibit C3 deposition onto the surface of cells leading to the inhibition of the classical complement pathway $(4,5)$. The structure of CSMD1 predicts that CSMD1 is a receptor for unknown ligand(s) and is involved in signal transduction (1).

CSMD1 is believed to act as a tumour suppressor based on a number of observations. CSMDI is located on chromosome 8p23, a region that is frequently deleted in different types of cancer (1,6-10). In addition, reduced CSMD1 mRNA and protein expression has been observed in different cancers (11-17). An array comparative genomic hybridization study revealed a high rate of loss of 8 p23.2 containing the CSMD1 gene in advanced prostrate cancer samples. A further small real-time PCR study identified a decrease in CSMD1 mRNA levels in higher stage prostrate cancer samples (11). Similarly reduced CSMD1 expression at the mRNA level has been identified in colorectal cancer associated with reduced patient survival (12). Furthermore, somatic mutations were detected in CSMD1 in $11 \%(6 / 54)$ of colorectal cancer patients along side DNA methylation and allele loss, predominantly in early onset patients (13). Previously, we demonstrated in a large series of breast cancers that 79/275 (28.7\%) had reduced CSMD1 protein expression. Low CSMD1 expression was significantly associated with high tumour grade $(\mathrm{p}=0.003)$ and decreased overall survival $(\mathrm{p}=0.018)$. Importantly multivariate analysis showed that CSMD1 was also an independent predictor of overall survival $(p=0.03)(14)$. 
Recently, in vivo and in vitro studies using the A375 melanoma cell line have also shown CSMD1 functions as a tumour suppressor gene (15). Overexpression of CSMD1 in melanoma cells resulted in reduced migration and proliferation and induced apoptosis. In addition, xenografted tumours expressing CSMDI resulted in reduction of tumour weight and size (15) Of note, reduced mRNA CSMD1 expression has been identified in glioblastoma stem cells compared to neural stem cells (16). Downregulation of CSMD1 was linked to upregulation of the microRNAs miR-10a and miRNA$10 \mathrm{~b}$ which formed an inhibitory complex with the 3'UTR of CSMD1 (16). Similarly high levels of miRNA-10b associated with low levels of CSMD1 expression were identified in HepG2 hepatocellular carcinoma cells (17).

In this study, we investigated the consequences of loss of CSMD1 expression on cell behaviour. We used a threedimensional (3D) MCF10A/Matrigel model to study the role of CSMD1 in mammary cell differentiation. This culture system has been recently used to address fundamental questions about processes that disrupt epithelial architecture. It provides culture conditions that allow mammary epithelial cells to respond to extracellular matrix (ECM) signals that impact upon proliferation, differentiation or death $(18,19)$. We found that loss of CSMD1 expression enhanced cell proliferation, migration and invasion. Furthermore, it reduced cell-adhesion. Moreover, in the 3D culture system, reduced CSMD1 expression disrupted the morphogenesis of epithelial structures and impaired lumen formation.

\section{Materials and methods}

Cell culture. MCF10A cells were obtained from the American Type Culture Collection and LNCaP cells were obtained from European Collection of Cell Cultures. MCF10A was cultured in DMEM-F12 medium (Invitrogen, Paisley, UK) as previously described (18). LNCaP cells were cultured in RPMI (Invitrogen) containing 10\% foetal calf serum (FCS) (Sigma-Aldrich, Dorset, UK). All cell lines were maintained at $37^{\circ} \mathrm{C}$ and $5 \% \mathrm{CO}_{2}$ in the tissue culture incubator and were routinely tested using MycoAlert ${ }^{\circledR}$ Mycoplasma detection assay (Lonza, Walkersville, MD, USA).

Generation of shCSMD1 stable cell lines. CSMD1 shRNA pRS vectors $\left(\mathrm{HuSH}^{\mathrm{TM}}\right.$, OriGene, Rockville, MD, USA) were transformed into NEB 5- $\alpha$ competent $E$. coli (New England BioLabs, Ipswich, MA, USA) and grown in L broth containing $50 \mu \mathrm{g} / \mathrm{ml}$ ampilicillin (Sigma-Aldrich). Sequences of CSMD1 shRNA constructs and their cognate CSMDI mRNA regions are shown in Table I. For each cell line, $1 \times 10^{6}$ cells $/ \mathrm{ml}$ were transfected with either shCSMD1 or shcontrol constructs using Lipofectamine ${ }^{\mathrm{TM}} 2000$ (Invitrogen). Cells were then grown in media that contained the appropriate dose $(0.5 \mu \mathrm{g} / \mathrm{ml}$, MCF10A; $0.01 \mu \mathrm{g} / \mathrm{ml}, \mathrm{LNCaP}$ ) of puromycin (Sigma-Aldrich) for 2 weeks. Single cell colonies were selected by serial dilutions in 96-well plates.

Reverse transcriptase polymerase chain reaction (RT-PCR). RNA was extracted from cells using TRIzol (Invitrogen). First strand cDNA synthesis was then performed using ThermoScript (Invitrogen) and random hexamers (Promega,
Southampton, UK) following the manufacturer's instructions. To test the specificity of shRNA constructs, CSMD3 was amplified. PCR reactions were performed using HotStarTaq (Qiagen, West Sussex, UK) following the manufacturer's instructions. The final extension step was for $10 \mathrm{~min}$ at $68^{\circ} \mathrm{C}$. Annealing temperature of the $C S M D 3$ primers was $52^{\circ} \mathrm{C}$ and $50^{\circ} \mathrm{C}$ for the housekeeping gene $R P L P O$. PCR products were analysed in $1.5 \%$ agarose gel containing ethidium bromide. For primer sequences of $C S M D 3$ and the housekeeping gene RPLPO, see Table II.

Quantitative real-time PCR reactions ( $q$ RT-PCR). CSMDI and $R P L P 0$ expression levels were investigated in all shCSMD1 and shcontrol clones using the relative standard curve method. TaqMan reactions, using sensiMix dT master mix (Quantace, London, UK), were performed in duplicates and run on an ABI7500 (Applied Biosystems, Warrington, UK). For sequences of primers and probes, see Table III.

Immunohistochemistry (IHC). IHC was performed to confirm the knockdown of CSMD1 protein expression in shRNA clones. Cells were pelleted, formalin fixed and paraffin-embedded and CSMD1 was stained using chicken anti-CSMD1 antibody at a 1:3000 dilution, as previously described (14). Negative controls, in which the pre-immune serum was applied, and positive controls of normal breast tissue were included in each batch of IHC.

Cell viability. Cell viability was evaluated using the MTT (3-(4,5-dimethylthiazol-2-yl)-2,5-diphenyltetrazolium bromide) assay. Cells $\left(1 \times 10^{4}\right.$ cells $\left./ \mathrm{ml}\right)$ were incubated with MTT solution $(1 \mathrm{mg} / \mathrm{ml})$ (Sigma-Aldrich) for $3-4 \mathrm{~h}$ at $37^{\circ} \mathrm{C}$. Crystals were dissolved in propan-1-ol and their optical densities (OD) were quantified at $570 \mathrm{~nm}$ (Opsys MR Plate reader; Dynex Technologies, West Sussex, UK).

Wound healing assay. Confluent monolayers were incubated in mitomycin C $(10 \mu \mathrm{g} / \mathrm{ml})$ (ICN Biomedicals, Costa Mesa, CA, USA) for $2 \mathrm{~h}$ at $37^{\circ} \mathrm{C}$ to inhibit cell division. Wounds were introduced into the monolayer by scratching with a P200 micropipette tip. Wound closure was followed for $96 \mathrm{~h}$ and phase contrast images were captured using an Olympus digital still camera attached to an Olympus inverted microscope with a $4 \mathrm{X}$ objective lens. The wound area was imaged using ImageJ and expressed as a percentage of the wound area covered at each time point relative to the surface area of the wound at time zero.

Transwell invasion assay. Invasion assays were carried out as previously described (20). The upper sides of 12-well format transwell inserts (BD Biosciences, Franklin Lakes, NJ, USA) were coated with Matrigel at a 1:10 dilution in serum-free medium (SFM). The undersides of the inserts were coated with fibronectin $(10 \mu \mathrm{g} / \mathrm{ml}$ in PBS). Medium containing FCS $(1 \mathrm{ml})$ was added to the base of each well. Cells at $5 \times 10^{4}$ cells $/ \mathrm{ml}$ in SFM were placed on the top of inserts, for $16 \mathrm{~h}$. Invasive cells that penetrated through to the underside of the inserts were identified by fixation in $4 \%$ paraformaldehyde and staining with crystal violet. Cells were then destained using $2 \%$ SDS and the OD was measured using the Opsys MR Plate reader at 
Table I. shCSMD1 construct sequences.

\begin{tabular}{llr} 
Construct & \multicolumn{1}{c}{ Sequence } & The cognate CSMD1 mRNA \\
\hline 1 & CCA CAG GCA GAA ATG CTT ACT GAG ATG A & $5234-5263 \mathrm{bp}$ \\
2 & GAG GAC ATC CAC AGC ACC TTC AAC TCA CT & $2505-2534 \mathrm{bp}$ \\
3 & GGC TTC CTC ATC CAC TAT GAG AGT GTG AC & $1013-1042 \mathrm{bp}$ \\
4 & CAT AGC CAT ACC TCT GAT GGA CAA GCA GT & $8929-8958 \mathrm{bp}$
\end{tabular}

$570 \mathrm{~nm}$. The percentage of invasion was calculated independently using the following equation: Cell invasion $(\%)=\mathrm{OD}$ of cells in the bottom/OD of cells in the bottom + OD of cells in the top.

Cell adhesion assays. Plates (96-well) were coated with fibronectin (10 $\mu \mathrm{g} / \mathrm{ml}$ in PBS) or Matrigel (20 $\mu \mathrm{g} / \mathrm{ml}$ in SFM). Non-specific adhesion sites were blocked by pre-incubation in $0.5 \%$ bovine serum albumin in DMEM or RPMI. Cells at $4 \times 10^{5}$ cells $/ \mathrm{ml}$ were pipetted onto the coated plates at $37^{\circ} \mathrm{C}$ and allowed to attach for $30 \mathrm{~min}$ or $2 \mathrm{~h}$. Following this, unattached cells were aspirated and the remaining attached cells were fixed in $4 \%$ paraformaldehyde and stained with crystal violet. Cells were then destained using $2 \%$ SDS and OD was measured using the Opsys MR Plate reader at $570 \mathrm{~nm}$.

MCF10A Matrigel assay. 3D Matrigel cultures of MCF10A cells were performed as previously described (18). Briefly, 8 -well glass slides (Nunc ${ }^{\mathrm{TM}}$; Nalge Nunc International Corp., Rochester, NY, USA) were coated with growth factor reduced Matrigel (BD Biosciences). shcontrol or shCSMD1 MCF10A cells (5000 cells/ml in 2\% Matrigel assay medium) were seeded onto the slides and incubated at $37^{\circ} \mathrm{C}$ for up to 26 days. Cells were fed every 3-4 days with 2\% Matrigel assay medium.

Fluorescence analysis and image acquisition. Fluorescent staining of acinar structures was performed as previously described (18). Phalloidin (Invitrogen), was used as a cytoskeletal marker at a 1:50 dilution. Rabbit polyclonal active caspase-3 antibody (Abcam, Cambridge, UK) was used as an apoptotic marker at a 1:100 dilution. Fluorescence staining was imaged using a Nikon confocal microscope (Nikon Eclipse TE2000-E; Nikon UK, Kingston Upon Thames, UK). Images of acinar structures were captured by serial confocal sectioning and viewed using confocal software. Phase contrast images were captured using an Olympus digital still camera attached to an Olympus inverted microscope (Olympus CKX41). All images were converted to TIFF format and figures were assembled and annotated using Adobe Photoshop 7.0 ${ }^{\circledR}$.

Statistical analysis. Initially the distribution of the data from all the functional assays except wound healing assay was tested using Shapiro-Wilk or Kolmogorov-Smirnov tests. When the data were normally distributed, a t-test was used to compare between means. A Mann-Whitney U test (non-parametric) was used when the data were not normally distributed. All tests were 2-sided and performed using SPSS software version 15. A p-value $\leq 0.05$ was considered to be statistically significant.
Table II. RT-PCR primer sequences.

\begin{tabular}{ll}
\hline Gene & \multicolumn{1}{c}{ Primers 5'-3' } \\
\hline CSMD3 & Forward: AGTAGTTCTGTAGCCATTGC \\
& Reverse: TGGGATCAAATCGTACCGCC \\
RPLPO & Forward: ACATGCTCAACATCTCCC \\
& Reverse: TTCAACCTTAGCTGGGG \\
\hline
\end{tabular}

\section{Results}

Generation of shCSMD1 stable cell lines. To help determine the function of CSMD1, shRNA gene silencing methodology was used to create stable cell lines with suppressed CSMD1 expression. Two different cell lines were analysed: MCF10A (normal breast) and LNCaP (prostate). Single cell colonies were selected from all cell lines and the mRNA expression level of CSMD1 was investigated using qRT-PCR. Colonies that showed the best level of mRNA knockdown were chosen for subsequent experiments. Two separate shCSMD1 cell lines were generated from the MCF10A cells (termed clone 1 and 2) and a single cell line was generated from the LNCaP cells. MCF10A shCSMD1 clones 1 and 2 showed a 66\% (SD $\pm 8.9 \%$ ) and $66.9 \%$ (SD $\pm 12 \%$ ) reduction in CSMD1 mRNA expression, respectively, when compared to shcontrol cells. The LNCaP cell line showed a $62 \%(\mathrm{SD} \pm 12 \%)$ CSMD1 mRNA knockdown, relative to shcontrols (Fig. 1A).

To confirm these levels of knockdown at the protein level, CSMD1 protein expression was investigated using IHC since at the time a CSMD1 antibody suitable for westerns was not available. MCF10A shCSMD1 colonies showed lower CSMD1 protein expression compared to the shcontrol. However, unexpectedly, the LNCaP shCSMD1 cells did not show a reduced level of protein expression compared to shcontrols cells in contrast to the high reduction in CSMDI mRNA expression observed by qRT-PCR (Fig. 1B).

To test the specificity of the shCSMD1 constructs the expression of CSMD3 mRNA was investigated using RT-PCR. Since normally none of the tested cell lines express CSMD2 its expression was not tested. The shCSMD1 constructs did not affect the expression pattern of CSMD3 in either of the cell lines examined. Moreover, the shcontrol plasmids had no effect on the expression pattern of CSMD3 (Fig. 1C).

Downregulation of CSMD1 disrupts cell morphology. To investigate the morphology of the shCSMD1 cells, the F-actin 

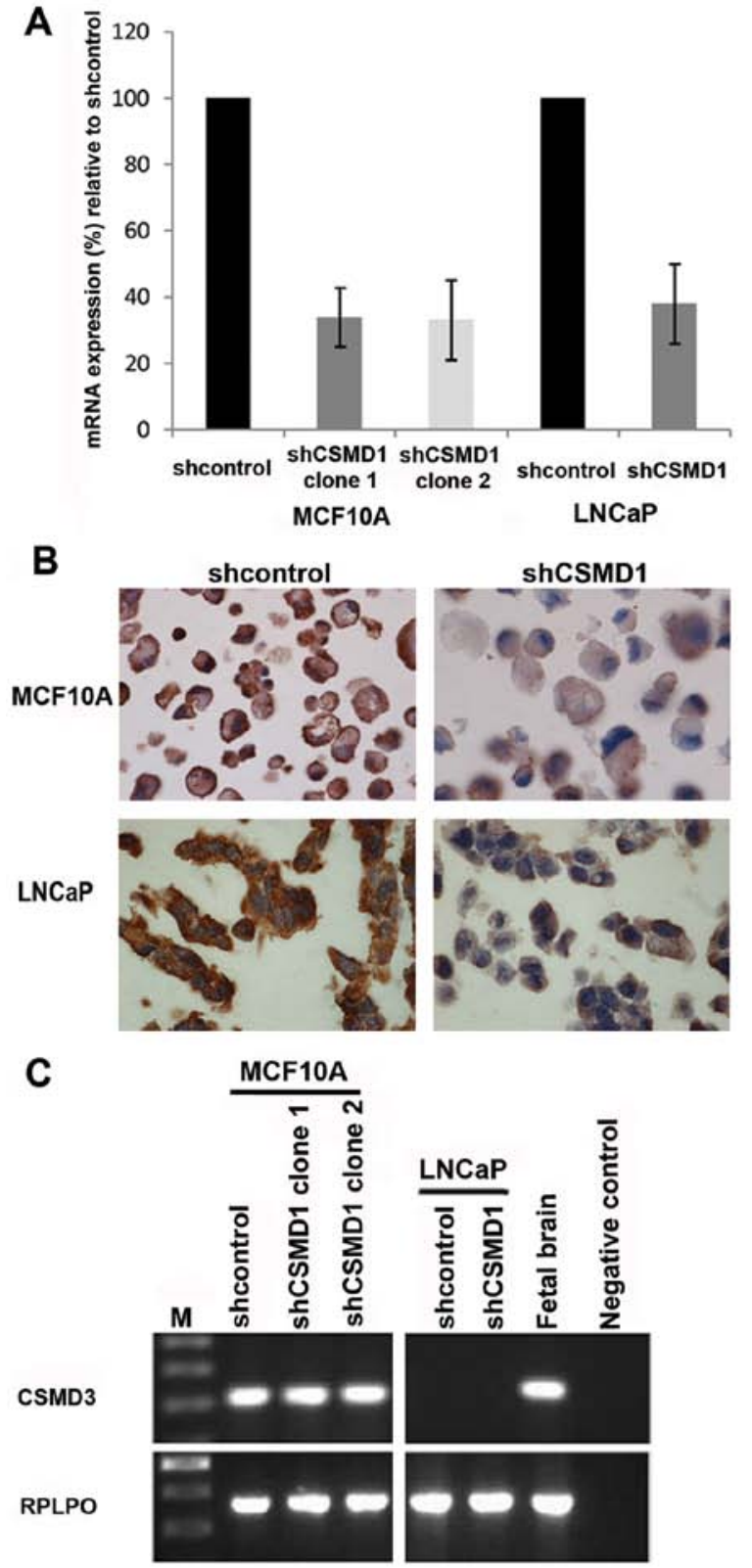

Figure 1. Confirmation of CSMD1 expression knockdown. (A) qRT-PCR, using the relative standard curve method confirmed the shRNA vectors reduced CSMD1 mRNA expression. MCF10A shCSMD1 clones 1 and 2, exhibited 66 and $67 \%$ reduction in CSMD1 expression, respectively. While, the knockdown efficiency in $\mathrm{LNCaP}$ was $62 \%$. The percentages of CSMD1 expression (relative to shcontrols) are presented as means \pm SD of at least 3 independent experiments. (B) IHC using chicken anti-CSMD1 antibody on formalin-fixed paraffin-embedded cells revealed that shCSMD1cells exhibit lower CSMD1 protein expression levels compared to shcontrols. Magnification, x100. (C) Confirmation of specificity of shRNA CSMD1 vectors. RT-PCR for CSMD3 in shCSMD1 cells did not detect any changes in the expression of this genes or the housekeeping gene RPLPO. Foetal brain cDNA was used as a positive control and water as a negative control. M, size standard marker.

stain Phalloidin was used to examine the cells. Staining revealed that in all lines examined, loss of CSMD1 expression resulted in misshapen cells that tended to grow individually. In contrast, shcontrol cells mainly grew as colonies. In MCF10A, many shCSMD1 cells possessed lamellipodia-like protrusions suggestive of a motile phenotype (Fig. 2, arrow). LNCaP shCSMD1 cells displayed very long filopodia-like protrusions

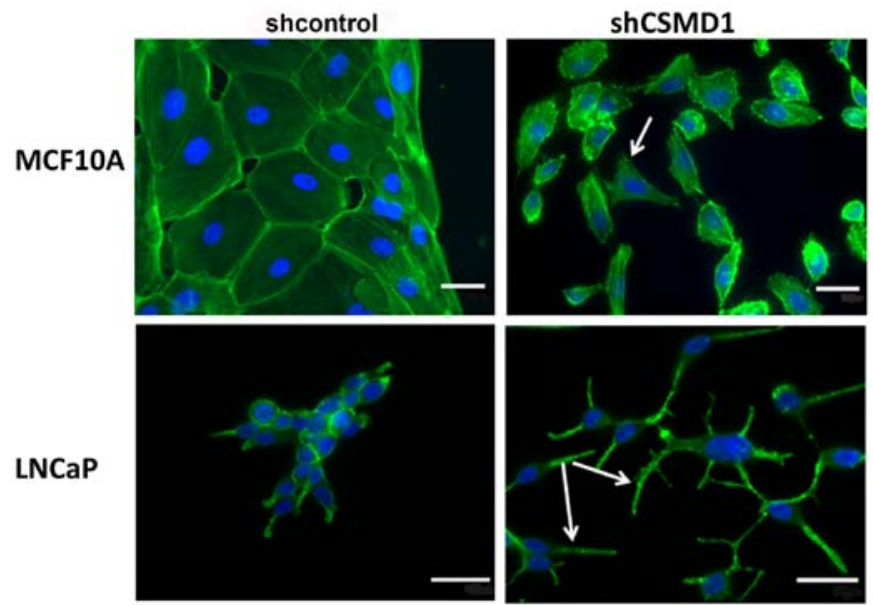

Figure 2. Loss of CSMD1 expression disrupts cell morphology. Phalloidin staining revealed that, in all cell lines, reduced CSMD1 expression resulted in misshapen cells lacking cell-cell contacts. Abundant MCF10A shCSMD1 cells are migrating with lamellipodia-like protrusions (arrow head). LNCaP shCSMD1 cells exhibit long filopodia-like protrusions (arrows). Nuclei are stained with DAPI. Magnification, x40; scale bar, $50 \mu \mathrm{m}$.

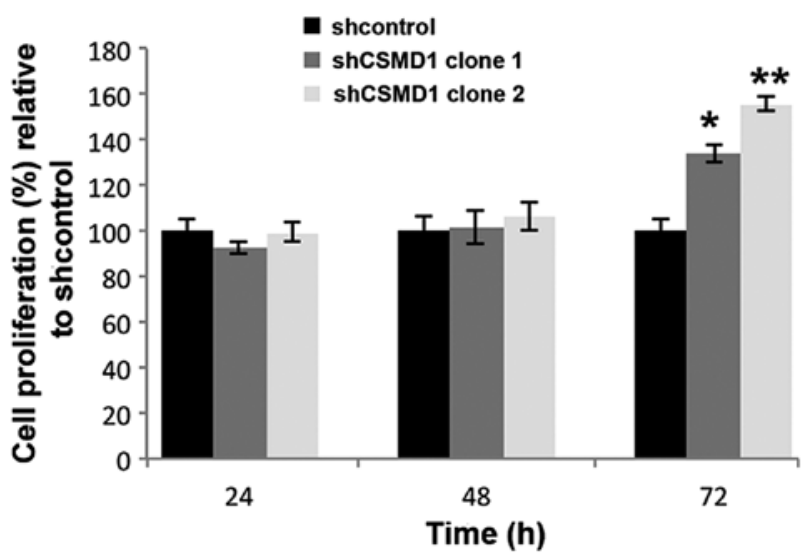

Figure 3. Loss of CSMD1 expression increases cell proliferation. MTT assays revealed that silencing of CSMD1 expression increased proliferation rates of MCF10A, after $72 \mathrm{~h}$, shCSMD1 clones 1 and 2 showed $34 \%\left({ }^{*} \mathrm{p}<0.003\right)$ and $56 \%\left({ }^{* *} \mathrm{p}<0.001\right)$ increase in proliferation. The percentages of cell proliferation, relative to shcontrols, are presented as the mean \pm SD of at least 3 independent experiments) after $24 \mathrm{~h}$, respectively.

(Fig. 2, arrows). In all cases these features were not observed in the appropriate shcontrol cell lines.

Effect of CSMD1 suppression on cell proliferation, migration and invasion. Having shown that CSMD1 silencing affected cell morphology, using MCF10A cells as a model we focused on understanding its functional effects by investigating a number of important cellular processes. Since minimal knockdown at the protein level was observed in the LNCap cell line further functional work was not performed in these cells. Cell proliferation was assayed using the MTT protocol. Silencing CSMD1 expression did not significantly affect cell proliferation until $72 \mathrm{~h}$ post-plating when increases of $34 \%$ (SD $\pm 4 \%$, $\mathrm{p}=0.003)$ and $56 \%(\mathrm{SD} \pm 3.3 \%, \mathrm{p}=0.001)$ were observed in MCF10A shCSMD1 clones 1 and 2 (Fig. 3).

Since the morphology of the MCF10A shCSMD1 cells suggested that they were more motile than their shcontrol 
Table III. qRT-PCR primer and probe sequences.

\begin{tabular}{lll}
\hline Gene & \multicolumn{1}{c}{ Primers } & \multicolumn{1}{c}{ Probe } \\
\hline CSMD1 & Forward: TTCCAGATTTTTATCCAAACTCTCTAA & CACGTGGACCATTGAAGTGTCTCATGG \\
& Reverse: GTGTGAAAGATCATTTGAACTCCTTT & \\
FPLPO & Forward: AGATGCAGCAGATCCGCAT & AGGCTGTGGTGCTGATGGGCAAGAAC \\
& Reverse: ATATGAGGAGCAGTTTCTCCAG & \\
\hline
\end{tabular}

A

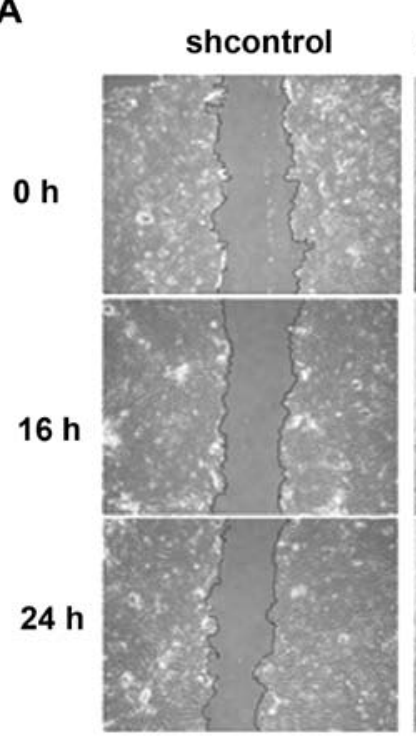

B

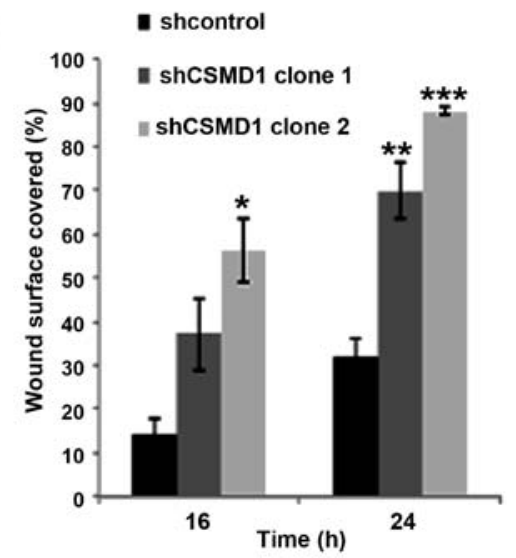

\section{shCSMD1 clone 1 shCSMD1 clone 2}
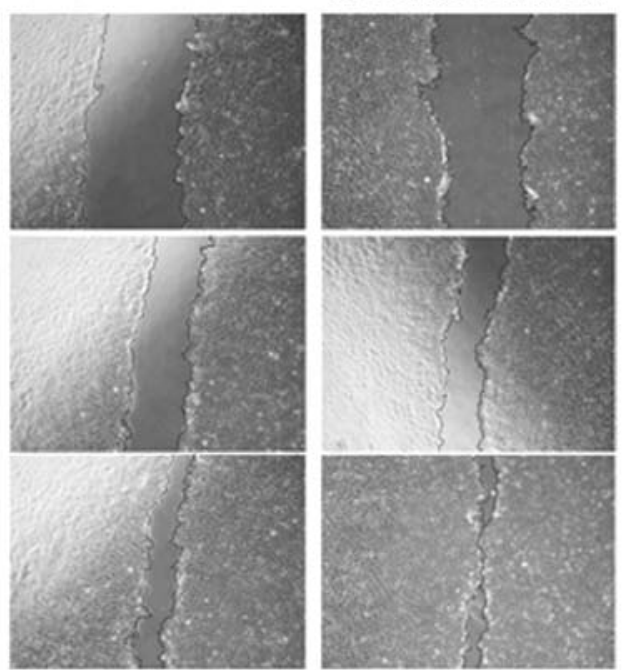

C

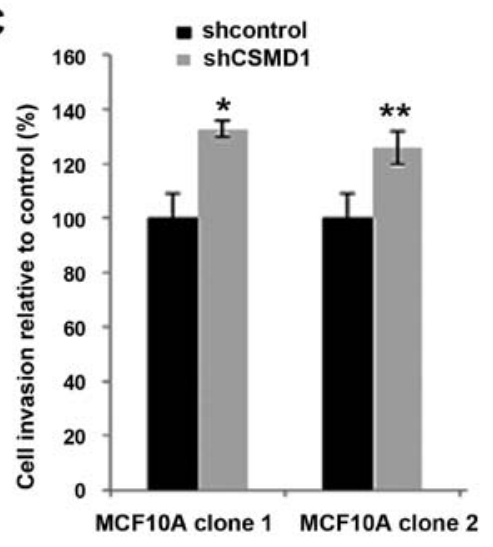

Figure 4. Loss of CSMD1 expression increases cell migration and invasion. (A) Wound healing assays, after inhibiting cell proliferation by mitomycin C, revealed that loss of CSMD1 expression enhanced migration of MCF10A cells. Images were taken, using phase contrast microscopy every $8 \mathrm{~h}$. Magnification, $\mathrm{x} 4$; scale bar, $500 \mu \mathrm{m}$. (B) In the MCF10A shcontrol cells 14.15 and $31.55 \%$ of the wound were covered after 16 and $24 \mathrm{~h}$, respectively. In the MCF10A shCSMD1 clones 1 and $2,37.02 \%(\mathrm{p}=0.07)$ and $56.15 \%(\mathrm{p}=0.018)$ of the wound were covered after $16 \mathrm{~h}$ and $69.9 \%\left({ }^{* *} \mathrm{p}=0.02\right)$ and $88.2 \%\left(^{* * *} \mathrm{p}=0.003\right)$ after $24 \mathrm{~h}, \mathrm{respec}-$ tively. The percentages of the surface area of the wound covered relative to time point zero, are presented as the mean \pm SD of two independent experiments. (C) MCF10A shCSMD1 clones 1 and 2 showed 33\% ( $\mathrm{p}<0.001)$ and $26 \%\left({ }^{* *} \mathrm{p}<0.001\right)$ increase in invasion, respectively. The percentages of cell invasion, relative to shcontrols, are presented as the mean $\pm \mathrm{SD}$ of at least 3 independent experiments.

counterparts (Fig. 2), we next investigated the role of CSMD1 in cell migration. Wound healing assays were performed after inhibiting MCF10A proliferation by treating cells with mitomycin $\mathrm{C}$ prior to the experiment. In the shcontrol cells $14.15 \%$ $(\mathrm{SD} \pm 3.6 \%)$ and $31.55 \%(\mathrm{SD} \pm 4.3 \%)$ of the wound were covered during the first $16 \mathrm{~h}$ and $24 \mathrm{~h}$ after wounding respectively. In the shCSMD1 clones 1 and 2, 37.02\% $(\mathrm{SD} \pm 8.5 \%, \mathrm{p}=0.07)$ and $56.15 \%(\mathrm{SD} \pm 7.1 \%, \mathrm{p}=0.018)$ of the wound were covered during the first $16 \mathrm{~h}$. Moreover, after $24 \mathrm{~h}$, cells in shCSMD1 clones 1 and 2 covered $69.9 \%(\mathrm{SD} \pm 6.6 \%, \mathrm{p}=0.02)$ and $88.2 \%$ $(\mathrm{SD} \pm 0.9 \%, \mathrm{p}=0.003)$ of the wound (Fig. $4 \mathrm{~A}$ and $\mathrm{B}$ ), confirming that suppression of CSMD1 expression promotes a more motile phenotype in MCF10A cells.

Since loss of CSMD1 promoted cell migration, we next studied its role in cell invasion. At $16 \mathrm{~h}$ after plating, suppression of CSMD1 expression resulted in $33 \%(\mathrm{SD} \pm 3 \%, \mathrm{p}<0.001)$ 


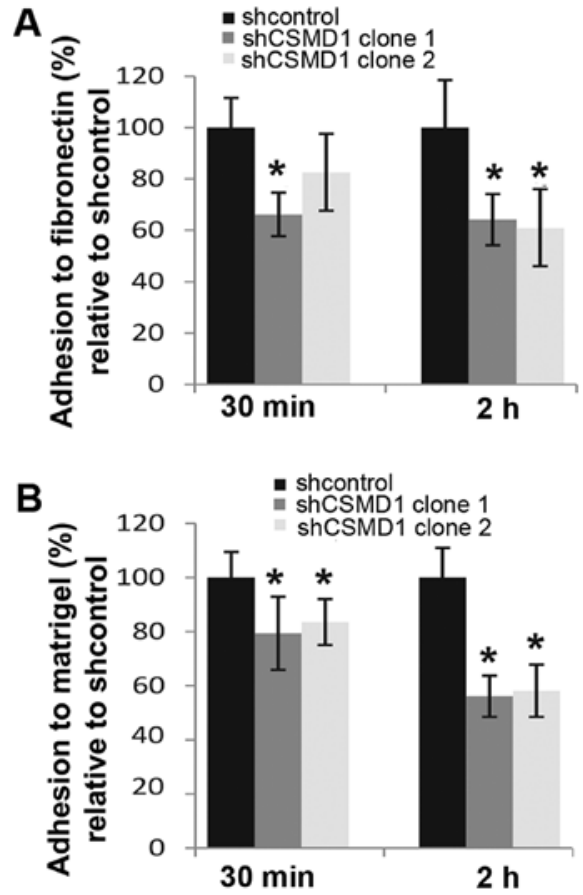

Figure 5. Loss of CSMD1 expression decreases cell adhesion to the ECM. Adhesion assays revealed that MCF10A shCSMD1 cell lines exhibit less adhesion to fibronectin (A) and Matrigel (B) than their shcontrols, at $30 \mathrm{~min}$ and $2 \mathrm{~h}$ post-cell plating. The percentages of adhesion, relative to shcontrols are presented as means \pm SD of at least 3 independent experiments. Asterisks (*) denote statistically significant differences between shcontrol cells and shCSMD1 clones ( $\left.{ }^{*} \mathrm{p}<0.05\right)$.

and $26 \%(\mathrm{SD} \pm 6 \%, \mathrm{p}<0.001)$ increases in cell invasion of MCF10A shCSMD1 clones 1 and 2, respectively, compared to shcontrol (Fig. 4C).

Effect of CSMD1 suppression on cell matrix adhesion. Shared homology with Cub domain containing protein 1 (CDCP1), which is involved in cell-matrix interactions $(21,22)$, suggested that CSMD1 could play a role in cell adhesion. To examine this possibility adhesion assays using Matrigel and fibronectin were performed $30 \mathrm{~min}$ and $2 \mathrm{~h}$ after cell plating. Loss of CSMD1 expression caused $\sim 34 \%$ (SD $\pm 8.5 \%$, $\mathrm{p}=0.001$ ) and $\sim 17 \%(\mathrm{SD} \pm 15 \%, \mathrm{p}=0.06)$ decreases in the adhesion of MCF10A shCSMD1 clones 1 and 2 to fibronectin after $30 \mathrm{~min}$, compared to shcontrol. After $2 \mathrm{~h}$, this difference had increased to $\sim 36 \%(\mathrm{SD} \pm 10 \%, \mathrm{p}=0.01)$ and $\sim 40 \%(\mathrm{SD} \pm 15 \%, \mathrm{p}=0.004)$ respectively (Fig. 5A). Following CSMD1 silencing, adhesion of MCF10A shCSMD1 clones 1 and 2 to Matrigel decreased by $20 \%(\mathrm{SD} \pm 13.5 \%, \mathrm{p}=0.02)$ and $16 \%(\mathrm{SD} \pm 8.5 \%, \mathrm{p}=0.05)$ after 30 min compared to shcontrol. Strikingly, $2 \mathrm{~h}$ after plating these differences had increased to $44 \%(\mathrm{SD} \pm 7.6 \%, \mathrm{p}=0.0006)$ and $\sim 42 \%$ ( $\mathrm{SD} \pm 9.6 \%, \mathrm{p}=0.001$ ) (Fig. $5 \mathrm{~B})$.

Loss of CSMD1 expression disrupts acini formation. We have previously observed that CSMD1 is highly expressed in welldifferentiated areas of breast cancer samples, suggesting that CSMD1 might play a role in mammary duct formation (14). To examine this further, an MCF10A 3D Matrigel model was established and the effects of CSMD1 knockdown on the morphogenesis of mammary acini were investigated. Loss of CSMD1 expression resulted in a larger number of acini and

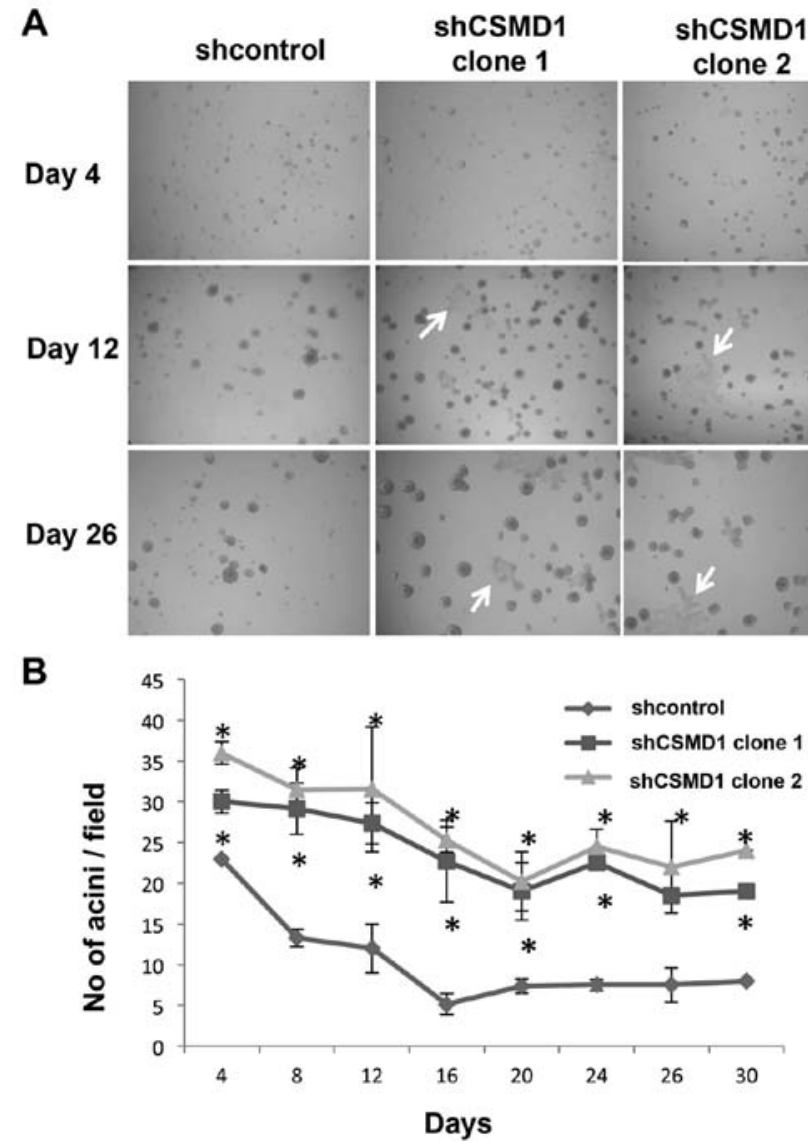

Figure 6. Reduced CSMD1 expression increases acini number and increases areas of flat cells in the MCF10A 3D model. (A) The morphogenesis of MCF10A acini was monitored and images were captured every 2-4 days, using phase contrast microscopy. Reduced CSMD1 expression caused an increase in the number of acini compared to shcontrol acini. shCSMD1 cells exhibited larger areas of flat cells compared to shcontrol cells (arrows). Magnification, $\mathrm{x} 4$; scale bar, $500 \mu \mathrm{m}$. (B) The numbers of MCF10A shCSMD1 acini are presented as the mean \pm SD of 3 fields from 3-5 independent experiments. Asterisks $(*)$ denote statistically significant differences between shcontrol cells and shCSMD1 clones (*p<0.05).

larger areas of flat cells (Fig. 6A, arrows). Acini in 3 fields from 3-5 independent experiments were counted and averaged every 2-4 days. There was a statistically significant increase in the average number of shCSMD1 acini compared to shcontrol at all time points $(\mathrm{p}<0.05)$ (Fig. 6B).

Phalloidin staining and confocal microscopy revealed that shCSMD1 acini were irregular in shape and heterogeneous in size (Fig. 7A). The diameter of each acinus was measured and the mean diameter of acini from shcontrol cells determined $(85 \mu \mathrm{m})$. This was then used as a cut-off to distinguish between large and small acini. Noteworthy, the proportion of large acini in MCF10A shCSMD1 clones 1 and 2 were $33 \%$ (SD $\pm 21 \%$, $\mathrm{p}=0.1)$ and $43 \%(\mathrm{SD} \pm 15 \%, \mathrm{p}=0.03)$ higher than in the shcontrol cell line (Fig. 7B). Furthermore, confocal imaging revealed that in shCSMD1 clones 1 and 2, respectively, 55\% (SD $\pm 9.8 \%$, $\mathrm{p}=0.045)$ and $90 \%(\mathrm{SD} \pm 8 \%, \mathrm{p}=0.008)$ of acini failed to form a lumen compared to shcontrol cells (Fig. 7C).

In this model system lumen formation depends on apoptosis, raising the possibility that a failure to generate acini with a lumen might be due to differences in developmentally regulated programmed cell death in cells where CSMD1 
A

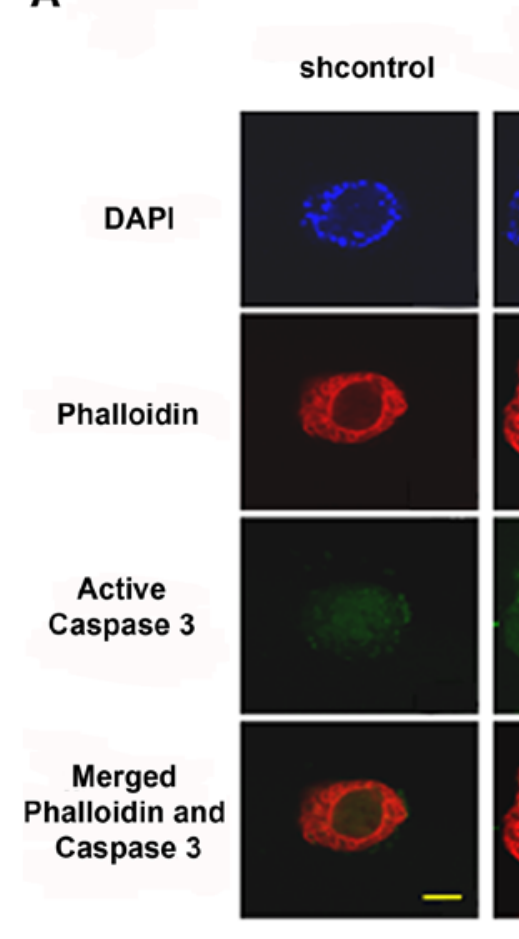

\begin{abstract}
shCSMD1 clone 1
\end{abstract}
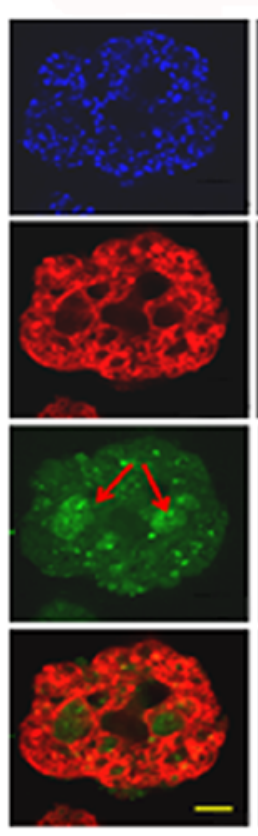

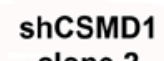

clone 2
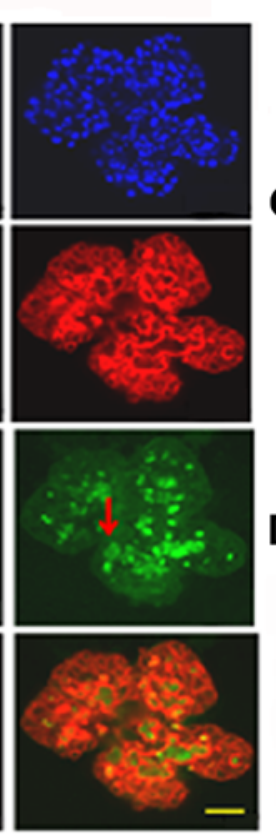
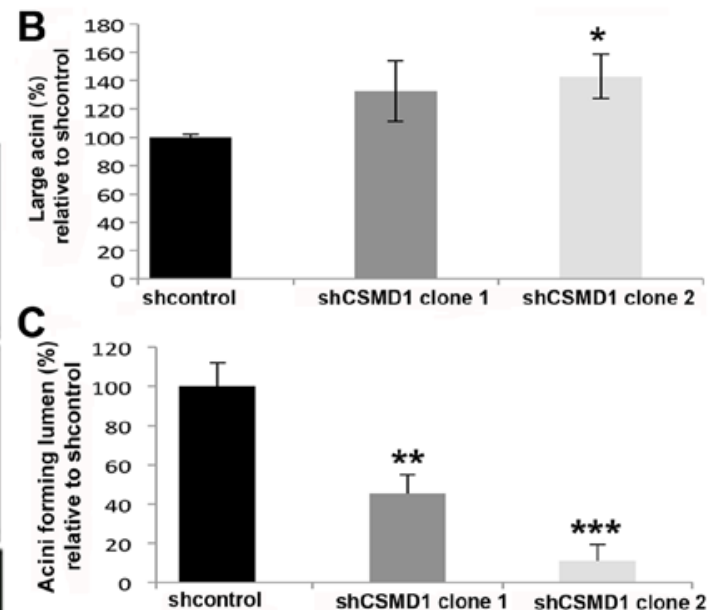

D

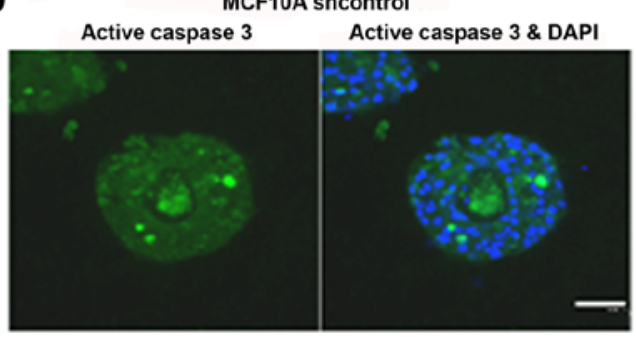

Figure 7. Silencing CSMD1 expression disrupts mammary acini morphology and inhibits lumen formation in the MCF10A 3D model. (A) Acini, at day 26, stained with DAPI (blue), phalloidin (red), and active caspase-3 antibody (green). Magnification, x40; scale bar, $50 \mu \mathrm{m}$. shCSMD1 acini are irregular in shape and heterogeneous in size with no lumen. shcontrol acini showed weak staining for active caspase-3, while, lumens of shCSMD1 acini exhibit strong staining (arrows). (B) The percentages of large acini, relative to shcontrol, are presented as the mean \pm SD of at least 3 independent experiments. Reduced CSMD1 expression resulted in $33 \%(\mathrm{p}=0.1)$ and $43 \%(\mathrm{p}=0.03)$ increase in the percentage of large acini in MCF10A shCSMD1 clones 1 and 2 , respectively. (C) The percentages of lumen forming acini, relative to shcontrol, are presented as the mean \pm SD of at least 3 independent experiments. Reduced CSMD1 expression resulted in $55 \%\left({ }^{* *} \mathrm{p}=0.045\right)$ and $90 \%\left({ }^{* * *} \mathrm{p}=0.008\right)$ decrease in the percentage of lumen forming acini in MCF10A shCSMD1 clones 1 and clone 2 , respectively. (D) shcontrol acini, at day 12 , stained with DAPI (blue) and active caspase-3 antibody (green). Magnification, $\mathrm{x} 40$; scale bar, $50 \mu \mathrm{m}$.

expression is inhibited. To test this, acini at day 12 (before the completion of lumen formation in shcontrol cells) and day 26 were stained with antibodies specific for active caspase-3. At day 26, lumens of shcontrol acini showed very weak staining compared to shCSMD1 lumens (Fig. 7A, arrows). However, at day 12, shcontrol acini showed strong staining of active caspase-3 (Fig. 7D). These observations confirm that during normal acinar development in this model system a lumen is generated by temporally coordinated apoptotic processes. Apoptosis is then severely suppressed or absent after the completion of lumen in shcontrol acini. In contrast, because shCSMD1 acini failed to form a lumen, apoptotic processes remain active until day 26 .

\section{Discussion}

CSMD1 encodes a transmembrane protein and is thought to function as a tumour suppressor. The function of CSMD1 is largely unknown. However, it is suggested to be a receptor or co-receptor involved in signal transduction (1). Herein, we investigated the biological consequences of loss of CSMD1 expression in cell line models. CSMD1 expression was suppressed in the cell lines MCF10A and LNCaP, using CSMD1 shRNA pRS vectors. The specificity of these vectors was validated, demonstrating that CSMD3 expression was not affected. This is an important consideration since it is possible that functional overlap between the CSMD proteins may modify the phenotype resulting from the loss of any one of them in tumours (1).

In both cell lines examined, downregulation of CSMD1 expression caused cell dissociation reflecting a more motile phenotype with enhanced formation of lamellipodia and filopodia-like protrusions. Formation of such structures is an indicator of extensive actin polymerisation (23). Previous studies have revealed that the rat ortholog of CSMD1 colocalizes with F-actin $(4,24)$. Thus CSMD1 may have an inhibitory effect on actin assembly or the signalling processes that impact upon the regulation of this process (25).

Further functional assays were focused on the MCF10A cell line which demonstrated high CSMD1 protein knockdown by IHC compared to the lower levels of protein knockdown observed in the LNCaP cells. Loss of CSMD1 expression increased cell proliferation. This agrees with studies that showed expression of the rat ortholog of CSMD1 is low in brain regions exhibiting high levels of cell proliferation (4). Similarly, a recent study has demonstrated that increasing miRNA-10b expression in HepG2 hepatocellular carcinoma cells resulted in decreased CSMD1 expression and increased proliferation (17). Our results also complement a study where increased expression of CSMD1 in A375 melanoma cells reduced proliferation (15). The inhibitory role of CSMD1 on cell proliferation is also in line with a potential role in 
cell differentiation, where CSMD1 enhances mammary duct formation. In normal development, growth arrest precedes differentiation and defects in the processes that control differentiation and proliferation are associated with carcinogenesis (26).

Reduced CSMD1 expression also enhanced cell migration in MCF10A CSMD1 knockdown cells. A similar increase in migration was identified in HepG2 hepatocellular carcinoma cells with increased miRNA-10b expression resulting in reduced CSMD1 expression (17). These findings also complement work in A375 melanoma cells where overexpression of CSMD1 reduced cell migration compared to control cells (15). Migration is a process of 4 steps; cell polarization, formation of lamellipodia, focal adhesions, and detachment of the cell rear as the cell front advances. Our data suggest that CSMD1 normally suppresses the formation of cellular morphologies associated with motility (such as lamellipodia). At focal adhesions integrins interact with actin providing a link between the cytoskeleton and the extracellular environment (27). The co-localisation of CSMD with both $\alpha 3$-integrin and F-actin suggests that CSMD1 may also be involved in the formation of these structures (24). Previous work has shown that the strength of focal adhesions determines the translocation velocity of the cell, with maximal velocities reached at intermediate adhesion strengths $(23,28-31)$. This might suggest that the presence of CSMD1 strengthens focal adhesions, which in turn impairs migration.

Since downregulation of CSMD1 expression affected cell migration, further investigations into whether changes in migration were concomitant with changes in cell invasion were undertaken by performing invasion assays using the shCSMD1 stable cell lines. Knock-down of CSMD1 expression significantly enhanced cell invasion of MCF10A cells. The inhibitory effect of CSMD1 on cell invasion agrees with work in HepG2 hepatocellular carcinomas cells where reduced expression of CSMD1 due to upregulation of miRNA-10b caused increased invasion (17). This study provides evidence of a role for CSMD1 in cell migration and invasion. However, the mechanisms underlying these roles have not been investigated. Together with its effects on cell morphology, it is interesting to speculate that the role of CSMD1 in migration and invasion is through its ability to modulate the cell cytoskeleton. This would be in agreement with a study by Tang et al in melanoma cells which demonstrated CSMD1 interacts with Smad3, activates Smad1, Smad2 and Smad3 and increase the expression of Smad4 (15). Smad3 has been shown to activate Rho signalling and cytoskeletal reorganisation (32).

Downregulation of CSMD1 expression in MCF10A cells decreased adhesion to Matrigel and fibronectin. The stimulatory role of CSMD1 in cell-matrix adhesion is consistent with the effects of other structurally similar proteins such as neuropilin-1 (33), bone morphogenetic protein 1 and TNF-stimulating gene $6(34,35)$ in this process.

In the 3D MCF10A model of duct formation, loss of CSMD1 expression resulted in a larger number of acini and an increase in the proportion of large acini. This may be due to the inhibitory effect of CSMD1 on cell proliferation. Moreover, shCSMD1 acini are irregular in shape, which may be attributed to the effect of CSMD1 on the morphology of individual cells. This finding is similar to a study that demonstrated distorted
MCF10A 3D structures as a result of overexpressed Akt or reduced expression of PDLIM2 (36,37).

Notably, a higher proportion of shCSMD1 acini failed to form a lumen. A filled in lumen is a hallmark feature of breast cancer/ductal carcinoma in situ. In breast development apoptosis plays a well-established role in lumen formation $(38,39)$. The MCF10A 3D model has been used extensively to investigate lumen formation in vitro and numerous studies have clearly demonstrated that MCF10A 3D acini failure to form a lumen is due to increased cell proliferation combined with inhibition of apoptosis $(18,36,40-42)$. In our model we show that this appears to be the case as caspase- 3 activity is observed in both shCSMD1 and shcontrol acini lumen at day 12. However, whereas in the shcontrol cells the expression of caspase-3 is reduced after the formation of the lumen, in the shCSMD1 acini caspase-3 expression is still strong and lumen formation was incomplete. It maybe that this is due to the increase in cell proliferation observed in cells with reduced CSMD1 expression. This idea agrees with studies suggesting that the maintenance of acinar architecture relies on the ability of increased cell death to counterbalance aberrant proliferation (18,36,40-42). Contrasting results were shown in a recent study in melanoma cell where CSMD1 overexpression resulted in reduced proliferation and increased apoptosis (15).

In future studies, it would be interesting to investigate mammary gland morphogenesis using an available CSMD1 knockout mouse model (43). Whole mounts of mouse fat pads would be prepared at $1,14,21,28,35$ days after parturition and the ductal network and terminal buds compared between Csmd1 (-/) and wild type (+/+) litter mates. IHC staining would also be performed for the proliferation marker Ki67 and apoptosis marker active caspase-3 to identify a potential regulatory imbalance between the two processes during mammary morphogenesis. Future studies would also address a limitation of the current study where CSMD1 protein knockdown was detected by IHC. Further experiments would involve the validation of the MCF10A knockdown cell lines by western blot using the newly available anti-CSMD1 antibody ab166908 from Abcam.

The effects of suppressing CSMD1 expression in our cell culture models suggest that CSMD1 may achieve its widespread effects on cell behaviour by playing a role in regulating cell morphology. Disruption of cell morphology affects processes as disparate as cell adhesion, proliferation, differentiation and motility (44-46). Cell morphology is mainly regulated by the actin cytoskeleton and CSMD1 has a potential role as a regulator for actin polymerisation via its potential interaction with F-actin. Furthermore, the broad effects of CSMD1 on cell functions may be due to its role in cell-ECM adhesion and its potential interaction with $\alpha 3$-integrin, which have similarly broad effects on the regulation of actin polymerization $(47,48)$, cell migration, formation of membrane protrusions (49), invasion and proliferation (50).

Loss of CSMD1 expression resulted in hallmarks of transformation including increased proliferation, migration and invasion. Our data support the proposed role of CSMD1 as a tumour suppressor. Potentially CSMD1 is involved in a signaling cascade regulating a wide range of cell processes. Future work will be aimed at dissecting the precise pathways involved. 


\section{Acknowledgements}

This study was supported by an Egyptian Government scholarship to M.K. and grants from Yorkshire Cancer Research grant number L292 (S.M.B.) and Breast Cancer Research Campaign grant numbers 2007NovPR53 (S.M.B. and V.S.) and 2008NovPR04 (D.H. and V.S.).

\section{References}

1. Sun PC, Uppaluri R, Schmidt AP, Pashia ME, Quant EC, Sunwoo JB, Gollin SM and Scholnick SB: Transcript map of the $8 \mathrm{p} 23$ putative tumor suppressor region. Genomics 75: 17-25, 2001.

2. Lau WL and Scholnick SB: Identification of two new members of the CSMD gene family. Genomics 82: 412-415, 2003.

3. Shimizu A, Asakawa S, Sasaki T, Yamazaki S, Yamagata H, Kudoh J, Minoshima S, Kondo I and Shimizu N: A novel giant gene CSMD3 encoding a protein with CUB and sushi multiple domains: A candidate gene for benign adult familial myoclonic epilepsy on human chromosome 8q23.3-q24.1. Biochem Biophys Res Commun 309: 143-154, 2003.

4. Kraus DM, Elliott GS, Chute H, Horan T, Pfenninger KH, Sanford SD, Foster S, Scully S, Welcher AA and Holers VM CSMD1 is a novel multiple domain complement-regulatory protein highly expressed in the central nervous system and epithelial tissues. J Immunol 176: 4419-4430, 2006.

5. Escudero-Esparza A, Kalchishkova N, Kurbasic E, Jiang WG and Blom AM: The novel complement inhibitor human CUB and Sushi multiple domains 1 (CSMD1) protein promotes factor I-mediated degradation of $\mathrm{C} 4 \mathrm{~b}$ and $\mathrm{C} 3 \mathrm{~b}$ and inhibits the membrane attack complex assembly. FASEB J 27: 5083-5093, 2013.

6. Toomes C, Jackson A, Maguire K, Wood J, Gollin S, Ishwad C, Paterson I, Prime S, Parkinson K, Bell S, et al: The presence of multiple regions of homozygous deletion at the CSMD1 locus in oral squamous cell carcinoma question the role of CSMD1 in head and neck carcinogenesis. Genes Chromosomes Cancer 37: 132-140, 2003

7. Macoska JA, Trybus TM, Benson PD, Sakr WA, Grignon DJ, Wojno KD, Pietruk T and Powell IJ: Evidence for three tumor suppressor gene loci on chromosome $8 \mathrm{p}$ in human prostate cancer. Cancer Res 55: 5390-5395, 1995.

8. Tørring N, Borre M, Sørensen KD, Andersen CL, Wiuf C and Ørntoft TF: Genome-wide analysis of allelic imbalance in prostate cancer using the Affymetrix 50K SNP mapping array. Br J Cancer 96: 499-506, 2007.

9. Blaveri E, Brewer JL, Roydasgupta R, Fridlyand J, DeVries S, Koppie T, Pejavar S, Mehta K, Carroll P, Simko JP, et al: Bladder cancer stage and outcome by array-based comparative genomic hybridization. Clin Cancer Res 11: 7012-7022, 2005.

10. Ma C, Quesnelle KM, Sparano A, Rao S, Park MS, Cohen MA, Wang Y,Samanta M,Kumar MS, Aziz MU, et al: Characterization of CSMD1 in a large set of primary lung, head and neck, breast and skin cancer tissues. Cancer Biol Ther 8: 907-916, 2009.

11. Paris PL, Andaya A, Fridlyand J, Jain AN, Weinberg V, Kowbel D, Brebner JH, Simko J, Watson JE, Volik S, et al: Whole genome scanning identifies genotypes associated with recurrence and metastasis in prostate tumors. Hum Mol Genet 13: 1303-1313, 2004.

12. Zhang R and Song C: Loss of CSMD1 or 2 may contribute to the poor prognosis of colorectal cancer patients. Tumour Biol 35 : 4419-4423, 2014.

13. Shull AY, Clendenning ML, Ghoshal-Gupta S, Farrell CL, Vangapandu HV, Dudas L, Wilkerson BJ and Buckhaults PJ: Somatic mutations, allele loss, and DNA methylation of the Cub and Sushi Multiple Domains 1 (CSMD1) gene reveals association with early age of diagnosis in colorectal cancer patients. PLoS One 8: e58731, 2013.

14. Kamal M, Shaaban AM, Zhang L, Walker C, Gray S, Thakker N, Toomes C, Speirs V and Bell SM: Loss of CSMD1 expression is associated with high tumour grade and poor survival in invasive ductal breast carcinoma. Breast Cancer Res Treat 121: 555-563, 2010.

15. Tang M-R, Wang Y-X, Guo S, Han S-Y and Wang D: CSMD1 exhibits antitumor activity in A375 melanoma cells through activation of the Smad pathway. Apoptosis 17: 927-937, 2012.
16. Lang M-F, Yang S, Zhao C, Sun G, Murai K, Wu X, Wang J, Gao H, Brown CE, Liu X, et al: Genome-wide profiling identified a set of miRNAs that are differentially expressed in glioblastoma stem cells and normal neural stem cells. PLoS One 7: e36248, 2012.

17. Zhu Q, Gong L, Wang J, Tu Q, Yao L, Zhang JR, Han XJ, Zhu SJ, Wang SM, Li YH, et al: miR-10b exerts oncogenic activity in human hepatocellular carcinoma cells by targeting expression of CUB and sushi multiple domains 1 (CSMD1). BMC Cancer 16: 806, 2016.

18. Debnath J, Muthuswamy SK and Brugge JS: Morphogenesis and oncogenesis of MCF-10A mammary epithelial acini grown in three-dimensional basement membrane cultures. Methods 30: 256-268, 2003

19. Shaw KR, Wrobel CN and Brugge JS: Use of three-dimensional basement membrane cultures to model oncogene-induced changes in mammary epithelial morphogenesis. J Mammary Gland Biol Neoplasia 9: 297-310, 2004.

20. Holliday DL, Hughes S, Shaw JA, Walker RA and Jones JL: Intrinsic genetic characteristics determine tumor-modifying capacity of fibroblasts: Matrix metalloproteinase-3 5A/5A genotype enhances breast cancer cell invasion. Breast Cancer Res 9: R67, 2007.

21. Scherl-Mostageer M, Sommergruber W, Abseher R, Hauptmann R, Ambros P, Schweifer $\mathrm{N}$ and Schweifer N: Identification of a novel gene, CDCP1, overexpressed in human colorectal cancer. Oncogene 20: 4402-4408, 2001.

22. Benes CH, Poulogiannis G, Cantley LC and Soltoff SP: The SRC-associated protein CUB Domain-Containing Protein-1 regulates adhesion and motility. Oncogene 31: 653-663, 2012.

23. Yilmaz M and Christofori G: EMT, the cytoskeleton, and cancer cell invasion. Cancer Metastasis Rev 28: 15-33, 2009.

24. Kraus DM, Pfenninger KH, Sanford SD and Holers VM: CSMD1 is expressed as a membrane protein on neuronal growth cones that colocalizes with F-actin and alpha-3 integrin. Mol Immunol 44: 198, 2007.

25. Ulrich F and Heisenberg C-P: Trafficking and cell migration. Traffic 10: 811-818, 2009.

26. Scott RE, Tzen CY, Witte MM, Blatti S and Wang H: Regulation of differentiation, proliferation and cancer suppressor activity. Int J Dev Biol 37: 67-74, 1993.

27. Ziober BL, Silverman SS Jr and Kramer RH: Adhesive mechanisms regulating invasion and metastasis in oral cancer. Crit Rev Oral Biol Med 12: 499-510, 2001.

28. Huttenlocher A, Sandborg RR and Horwitz AF: Adhesion in cell migration. Curr Opin Cell Biol 7: 697-706, 1995.

29. Lambrechts A, Van Troys M and Ampe C: The actin cytoskeleton in normal and pathological cell motility. Int J Biochem Cell Biol 36: 1890-1909, 2004.

30. Palecek SP, Huttenlocher A, Horwitz AF and Lauffenburger DA: Physical and biochemical regulation of integrin release during rear detachment of migrating cells. J Cell Sci 111: 929-940, 1998.

31. Cox EA, Sastry SK and Huttenlocher A: Integrin-mediated adhesion regulates cell polarity and membrane protrusion through the Rho family of GTPases. Mol Biol Cell 12: 265-277, 2001.

32. Lee J, Moon HJ, Lee JM and Joo CK: Smad3 regulates Rho signaling via NET1 in the transforming growth factor-betainduced epithelial-mesenchymal transition of human retinal pigment epithelial cells. J Biol Chem 285: 26618-26627, 2010

33. Valdembri D, Caswell PT, Anderson KI, Schwarz JP, König I, Astanina E, Caccavari F, Norman JC, Humphries MJ, Bussolino F, et al: Neuropilin-1/GIPC1 signaling regulates alpha5beta1 integrin traffic and function in endothelial cells. PLoS Biol 7: e25, 2009.

34. Huang G, Zhang Y, Kim B, Ge G, Annis DS, Mosher DF and Greenspan DS: Fibronectin binds and enhances the activity of bone morphogenetic protein 1. J Biol Chem 284: 25879-25888, 2009.

35. Kuznetsova SA, Mahoney DJ, Martin-Manso G, Ali T, Nentwich HA, Sipes JM, Zeng B, Vogel T, Day AJ and Roberts DD: TSG-6 binds via its CUB_C domain to the cellbinding domain of fibronectin and increases fibronectin matrix assembly. Matrix Biol 27: 201-210, 2008.

36. Debnath J, Walker SJ and Brugge JS: Akt activation disrupts mammary acinar architecture and enhances proliferation in an mTOR-dependent manner. J Cell Biol 163: 315-326, 2003.

37. Deevi RK, Cox OT and O'Connor R: Essential function for PDLIM2 in cell polarization in three-dimensional cultures by feedback regulation of the $\beta 1$-integrin-RhoA signaling axis. Neoplasia 16: 422-431, 2014. 
38. Frisch SM and Francis H: Disruption of epithelial cell-matrix interactions induces apoptosis. J Cell Biol 124: 619-626, 1994.

39. Humphreys RC, Krajewska M, Krnacik S, Jaeger R, Weiher H, Krajewski S, Reed JC and Rosen JM: Apoptosis in the terminal endbud of the murine mammary gland: A mechanism of ducta morphogenesis. Development 122: 4013-4022, 1996.

40. Debnath J, Mills KR, Collins NL, Reginato MJ, Muthuswamy SK and Brugge JS: The role of apoptosis in creating and maintaining luminal space within normal and oncogene-expressing mammary acini. Cell 111: 29-40, 2002.

41. Hebner C, Weaver VM and Debnath J: Modeling morphogenesis and oncogenesis in three-dimensional breast epithelial cultures. Annu Rev Pathol 3: 313-339, 2008.

42. Yanochko GM and Eckhart W: Type I insulin-like growth factor receptor over-expression induces proliferation and anti-apoptotic signaling in a three-dimensional culture model of breast epithelial cells. Breast Cancer Res 8: R18, 2006.

43. Steen VM, Nepal C, Ersland KM, Holdhus R, Nævdal M, Ratvik SM, Skrede S and Håvik B: Neuropsychological deficits in mice depleted of the schizophrenia susceptibility gene CSMD1. PLoS One 8: e79501, 2013.
44. Boivin D, Bilodeau D and Béliveau R: Regulation of cytoskeletal functions by Rho small GTP-binding proteins in normal and cancer cells. Can J Physiol Pharmacol 74: 801-810, 1996.

45. Rao KM and Cohen HJ: Actin cytoskeletal network in aging and cancer. Mutat Res 256: 139-148, 1991.

46. Trump BF, Heatfield BM, Phelps PC, Sanefuji H and Shamsuddin AK: Cell surface changes in preneoplastic and neoplastic epithelium. Scan Electron Microsc 3: 43-60, 1980.

47. Vermeulen K, Van Bockstaele DR and Berneman ZN: The cell cycle: A review of regulation, deregulation and therapeutic targets in cancer. Cell Prolif 36: 131-149, 2003.

48. Kharitonova MA and Vasiliev JM: Controlling cell length. Semin Cell Dev Biol 19: 480-484, 2008.

49. Frame MC and Brunton VG: Advances in Rho-dependent actin regulation and oncogenic transformation. Curr Opin Genet Dev 12: 36-43, 2002.

50. Alexandrova AY: Evolution of cell interactions with extracellular matrix during carcinogenesis. Biochemistry (Mosc) 73: 733-741, 2008. 